\title{
THE UNIVERSITY STUDENT TRAVEL MARKET: MOTIVATIONS AND PREFERENCES FOR ACTIVITIES
}

\author{
HUAN XIAO, ${ }^{* 1}$ KEVIN KAM FUNG SO,$\dagger^{1}$ AND YING WANG*1 \\ *Department of Tourism, Sport and Hotel Management, Griffith Business School, \\ Griffith University, Gold Coast, Queensland, Australia \\ †School of Hotel, Restaurant and Tourism Management, Center of Economic Excellence in \\ Tourism and Economic Development, University of South Carolina, Columbia, SC, USA
}

\begin{abstract}
Despite the significance of the student travel market, this segment's heterogeneity in motivation and behavior remains relatively unexplored. This article presents a comparative analysis of domestic and international university students' travel motivations and activity preferences. Using Partial Least Squares Path Modeling, questionnaire data from 307 students showed that students were most likely to seek opportunities for learning and relaxation. Further, the student market lacks homogeneity in terms of travel motivation and preference for activities, and motivational factors are significantly associated with activity preference. The study provides empirical support for the motivation-behavior link in preferences for activities, and offers insights into students' travel motivation and needs, enabling more effective product design and marketing for this important segment. Differences between domestic and international students suggest a need for differentiated strategies for the two groups of travelers.
\end{abstract}

Key words: University students; Motivation; Tourist activity; Australia

Introduction

University students represent an important segment for the tourism and leisure sectors (K. Kim, Oh, \& Jogaratnam, 2007). Their relatively low daily expenditure is generally compensated for by a longer length of stay (Y. Wang \& Davidson, 2008), and they travel more frequently and engage in more tourist experiences than might have been expected (Richards \&
Wilson, 2004), thus raising this segment's potential to be a viable target market. In addition, student travelers should be studied as a discrete market segment as they substantially differ from the youth market in terms of motivations, travel patterns, and preferences (Pearce \& Son, 2004; Richards \& Wilson, 2004). For instance, Pearce and Son (2004) found that compared to backpackers, international English language student travelers were more likely to travel in a large group, stay

${ }^{1}$ Authors contributed equally to this article.

Address correspondence to Kevin Kam Fung So, School of Hotel, Restaurant and Tourism Management, Center of Economic Excellence in Tourism and Economic Development, University of South Carolina, Columbia, SC 29208, USA. E-mail: kevinso@hrsm.sc.edu 
in hotel or motel, and be interested in city-based travel activities. Further, the student travel industry has a clearly defined structure and clientele (Richards \& Wilson, 2004). However, this segment is often subsumed within the wider youth travel market and has received little dedicated academic or market attention (K. Kim et al., 2007; Richards \& Wilson, 2004; X. Wang \& Walker, 2010), although students have often conveniently served as pilot samples in larger studies. Researchers therefore call for a deeper understanding of this segment (e.g., Richards \& Wilson, 2004; Sakakida, Cole, \& Card, 2004; X. Wang \& Walker, 2010; Xu, Morgan, \& Song, 2009).

University students are a heterogeneous group, requiring examination in a cross-country context (K. Kim \& Jogaratnam, 2003; Shoham, Schrage, \& van Eeden, 2005; X. Wang \& Walker, 2010; Xu et al., 2009). The few studies relevant to Australia have focused on international students and have covered a limited range of topics such as contribution to tourism or destination perceptions (Y. Wang \& Davidson, 2008; Weaver, 2003). The literature has largely ignored Australian domestic students ( $72 \%$ of total student enrollments), who amount to more than twice the number of international students (28\%) in the higher education sector (Australian Bureau of Statistics, 2012). Differences between the two groups warrant exploration.

Furthermore, studies of tourist motivation and its behavioral consequences have concentrated on destination choice and paid little attention to motivation's influence on activity preference. Research on preferences for activities is important from a practical point of view, because it informs tourism and recreational planners not only of their customers' preferences, but also about the activities themselves and the physical environment in which these activities take place (Jackson \& Schinkel, 1981). For marketing, knowledge of activity preferences facilitates effective promotion (Law, Cheung, \& Lo, 2004) and allows the industry to pursue activity-based market segmentation (Hsieh, O’Leary, \& Morrison, 1992). Although the importance of activity preference in market segmentation and understanding recreation behavior is well established in the recreation literature (Law et al., 2004; Moscardo, Morrison, Pearce, Lang, \& O’Leary, 1996), it is underappreciated in the field of tourism.

This study compares the travel motivations and activity preferences of international and domestic students in Australia. Australia has been chosen because of its sizable international student market (Australian Bureau of Statistics, 2012) and the tourism industry's significant impact on the economy (Hooper \& van Zyl, 2011). This study’s contribution is its comparative analysis of domestic and international students and its linking of travel motivational factors to activity preferences. In practical terms, this study seeks to provide a better understanding of how preferences for various types of activities reflect students' varied travel motivations, and to determine which customized itineraries and products the tourism sector needs to offer to more effectively reach this market.

\section{Literature Review}

Travel Motivation and Preference for Tourism Activity

Motivation is "an internal factor that arouses, directs, and integrates a person's behavior" (Murray, 1964, p. 7) and is a critical variable in explaining travel behavior (Crompton, 1979). In examining the influence of tourism motivation on destination choice, studies have employed the push-pull model (Crompton, 1979; Josiam, Smeaton, \& Clements, 1999; Mazzarol \& Soutar, 2002; Tangeland, Vennesland, \& Nybakk, 2012). Push factors refer to inner, intrinsic aspects such as anomie and ego enhancement, while pull factors relate to destination attributes (Dann, 1977). Once push motivations have been triggered, the tourist's focus shifts from the impetus dimension of motivation to the directive dimension, leading to the selection of a particular destination to fulfill the impetus motivations (Crompton, 1979).

Prior research (e.g., Kleiven, 2005; Ryan \& Glendon, 1998) has also relied on the leisure motivation theory, which disaggregates motivation to participate in leisure into four independent components (Beard \& Ragheb, 1983):

- the intellectual component (the extent to which individuals desire opportunities for learning, creating, exploring, discovering, or imagining);

- the social component (the need for the esteem of others and the need for friendship and interpersonal relationships); 
- the competence/mastery component (the need to achieve, master, challenge, and compete);

- the stimulus/avoidance component (the need to escape from a stressful everyday environment to rest and relax).

The leisure motivation theory is appropriate in the tourism context as the connection between leisure and tourism research has long been acknowledged (Smith \& Godbey, 1991), indicating the relevance of leisure knowledge to tourism phenomenon. In addition, the theory has been successfully applied in varying tourism contexts (Kleiven, 2005; Lounsbury \& Franz, 1990; Ryan \& Glendon, 1998) and its four components resonate with specific tourism motivation theories (Ryan \& Glendon, 1998). Finally, the theory's activity-oriented nature makes it suitable for this study's examination of tourism activities.

Studies of motivation focus largely on behavioral consequences such as its influence on destination choice (e.g., Josiam et al., 1999; Mansfeld, 1992; Mazzarol \& Soutar, 2002). As critical attributes of destinations, tourism activities are the main components of leisure trips. Tourism activities have previously received little academic attention, although some studies include activities as elements of the destination's image that pull tourists toward the destination (e.g., Tapachai \& Waryszak, 2000; Y. Wang \& Davidson, 2009). From this perspective, activities are motivating factors of the destination rather than behavioral consequences of motivation. Similarly, specialist activities are understood as factors driving participation in special interest tourism at a particular destination (Trauer, 2006).

The activity-based model of destination choice suggests that motivation affects the selection of activities and that tourists will choose destinations that offer their preferred activities (Moscardo et al., 1996). This model not only highlights the importance of activities in directing destination choice, but also indicates a direct relationship between travel motivation and activity, for example, among festival attendees (K. Kim, Sun, \& Mahoney, 2008) or with respect to the positive effect of the risk-taking motive on the purchase of nature-based tourism products (Tangeland et al., 2012).

The preceding review makes apparent the need to examine the influence of motivation on choice of tourism activities, which leads to our first three research questions:

RQ1: What are university students' motivations to undertake travel?

RQ2: What tourist activities do university students prefer to engage in while traveling?

RQ3: Does travel motivation influence students' preferences for activities?

While literature suggests a relationship between motivation and choice of tourism activities, this relationship has rarely been empirically tested, particularly in the student travel market. Therefore, more specifically related to RQ3 and based on Beard and Ragheb's (1983) four-motivation model, this study proposes that:

H1a: Stronger intellectual motivation is associated with higher preferences for activities providing opportunities for learning, creating, exploring, discovering, or imagining.

H1b: Stronger social motivation is associated with higher preferences for activities satisfying students' need for esteem, friendship, and interpersonal relationships.

H1c: Stronger competence/mastery motivation is associated with higher preferences for activities offering opportunities to achieve, master, challenge, and compete.

H1d: Stronger stimulus/avoidance motivation is associated with higher preferences for activities providing opportunities to escape from a stressful everyday environment to rest and relax.

\section{Student Travel Behaviors}

The cultural background of the traveler is known to influence travel motivation and behavior. For example, C. Kim and Lee (2000) found that American travelers show more individualistic characteristics whereas their Japanese counterparts exhibit more collectivistic characteristics. The two groups of travelers also differed in their motivations to seek prestige/status, family togetherness, and novelty. Similarly, differences in culture and values lead to a variety of travel preferences among university students. Japanese student travelers are more collectivistic, psychocentric, and likely to visit popular 
destinations than American students (Sakakida et al., 2004). A comparative study of students from the US, South Africa, and Israel revealed differences in choices of transportation, food, leisure activity, and accommodation (Shoham et al., 2005). Several studies also demonstrated differences in student travel behavior such as destination preference, use of travel services, and motivations (Field, 1999; X. Wang \& Walker, 2010; Xu et al., 2009).

Although individualism is high in British-influenced countries such as Australia (C. Kim \& Lee, 2000), the majority of Australia's international students have an Asian background (Australian Bureau of Statistics, 2012), which generally includes a strong tendency toward collectivism (C. Kim \& Lee, 2000). International and domestic students are thus likely to differ in terms of travel motivations and preferences for activities, leading to our fourth research question, as well as hypotheses $2 \mathrm{a}$ and $2 \mathrm{~b}$ :

RQ4: Do international students differ from domestic students in terms of motivations and preferred activities?

H2a: International and domestic students differ in their travel motivations.

H2b: International and domestic students differ in their preferred activities.

\section{Research Methods}

This study adopted a quantitative method. The leisure motivation theory provides a useful framework, with a corresponding 32-item measurement scale (Beard \& Ragheb, 1983). The scale’s statements and anchors were reworded to suit this study. The statements start with "one of my reasons for engaging in a leisure trip (within Australia) is . .." and respondents indicated their agreement with each statement on a 7-point scale ( 1 = never true, 7 = always true $)$.

The initial list of tourism activities was drawn from the Australian National Visitor Survey (NVS) and International Visitor Survey (IVS), both administered annually by Tourism Research Australia. Thirty-five activities were extracted; respondents were asked to indicate how likely they are to engage in each activity during a hypothetical leisure trip on a 7-point Likert scale ( 1 = very unlikely, 7 = very likely).

The questionnaire also collected information about gender, age, nationality, marital status, source of funds, preferred accommodation type, and other trip-related behaviors. International students were also asked to rate their confidence in communicating in English.

To elicit feedback on the questionnaire design, inclusiveness of items, clarity, and layout, the questionnaire was pretested with a convenience sample of international students $(n=11)$ and Australian students $(n=13)$. Responses led to slight modification of item wording to improve clarity. This study drew a convenience sample from two main campuses of a large Australian university that hosts over 43,000 students of which more than $25 \%$ are international from over 150 countries. Over 3 weeks, the data were collected through a self-administered questionnaire in public areas such as the libraries and cafeterias where most students gather. Students were approached for their voluntary assistance, and upon agreement to participate in the research respondents were given a project information sheet and a questionnaire to complete on the spot. In total, 307 usable responses were collected.

After an exploratory factor analysis (EFA) to evaluate the underlying structure of the measured variables, a series of independent samples $t$ tests were conducted to identify differences related to motivations and tourism activities between domestic and international students. Finally, Partial Least Squares Path Modeling (PLS-PM) analysis was performed to examine the links between motivations and tourism activities.

\section{Results}

The sample was reasonably equally distributed across genders and between international and domestic student groups (see Table 1). The average age of international students (24.3 years) is slightly higher than that of domestic students (20.6 years), and about $60 \%$ of respondents were single. Undergraduate students made up $76.8 \%$, compared to $21.2 \%$ postgraduates and 2\% English language students. Approximately 33\% of international participants were postgraduates as opposed to only $9.1 \%$ for the domestic group. In addition, the majority $(84.7 \%)$ of the international respondents were from an Asian country, with over half of them from China (50.7\%). More than $37 \%$ of the international 
Table 1

Sample Profile

\begin{tabular}{lccc}
\hline Characteristics & International $[N(\%)]$ & Australian $[N(\%)]$ & Total \\
\hline Gender & & & \\
$\quad$ Female & $74(48.7 \%)$ & $73(47.7 \%)$ & $147(48.2 \%)$ \\
$\quad$ Male & $78(51.3 \%)$ & $80(52.3 \%)$ & $158(51.8 \%)$ \\
$\quad$ Total & $152(100 \%)$ & $153(100 \%)$ & $305(100 \%)$ \\
Age & & & \\
$\quad$ Average age & $24.29(n=146)$ & $20.62(n=151)$ & $22.13(n=299)$ \\
Education & $96(63.2 \%)$ & $136(88.9 \%)$ & $232(76.8 \%)$ \\
$\quad$ Undergraduate & $50(32.9 \%)$ & $14(9.1 \%)$ & $64(21.2 \%)$ \\
$\quad$ Postgraduate & $4(2.6 \%)$ & $2(1.3 \%)$ & $6(2 \%)$ \\
$\quad$ English course & $150(100 \%)$ & $152(100 \%)$ & $302(100 \%)$ \\
$\quad$ Total & & & \\
Relationship status & $93(61.2 \%)$ & $87(57.2 \%)$ & $180(59.2 \%)$ \\
$\quad$ Single & $59(38.8 \%)$ & $65(42.8 \%)$ & $124(40.8 \%)$ \\
In a relationship & $152(100 \%)$ & $152(100 \%)$ & $304(100 \%)$ \\
$\quad$ Total & &
\end{tabular}

students felt confident or very confident of their English language skills.

To generate clean data for subsequent analysis, each component of Beard and Ragheb's (1983) motivation scale (i.e., intellectual, social, competence/mastery, and stimulus/avoidance) was analyzed in EFA using a principal component extraction method and varimax rotation. Items exhibiting interitem correlation coefficients of below 0.3 or over 0.9 or yielding factor loadings of less than 0.40 were removed (Hair, Black, Babin, Anderson, \& Tatham, 2006; Tabachnick \& Fidell, 2001).

As the activity scale was newly devised for this study, an EFA was conducted to explore the underlying structure of the data using principal axis extraction with direct oblimin rotation. Sixteen items were removed owing to factor loadings of below 0.4 or a cross-loading problem. Eventually, a clear solution was identified based on the eigenvalue greater than 1 criterion and Cattell's scree plot, with 19 items loading on four factors (see Table 2). The four factors were named Australian Identity (activities relating to Australia and its history and culture), Physical/Outdoor, Pleasure, and Nature-based, corresponding to Beard and Ragheb's (1983) four motivations.

Table 3 summarizes the results regarding travel motivations for both the overall sample and the two subsamples of international and domestic students. International students rated motivations higher than their domestic counterparts on 22 of the 32 items. Four items have a mean over 5 (often true), namely "to relax mentally," "to relieve stress and tension," "to rest," and "to discover new things," which were also the top four items for both the international students and the Australian students. The independent samples' $t$ test results indicate significant differences on 11 items that mainly concern learning, experiencing new things, and social motives.

Using the items retained after the EFAs, at the factor level, stimulus/avoidance was found the most motivating factor for all respondents, while competence/mastery was the least motivating. For Australians, the social factor was less motivating than competence/mastery, but the opposite was evident for the international respondents. The two groups also differed with respect to the intellectual factor and the social factor. This result is generally consistent with the pattern at the individual item level.

Table 4 shows that international students differed significantly from domestic students on 16 activities, mostly associated with Australian history, as well as its aboriginal and sport/outdoor cultures. Overall, the independent samples' $t$ test results at the factor level corresponded to item level results. More specifically, the pleasure factor had the highest mean (5.34), while Australian identity had the lowest (4.16). Despite the differences at the item level, only one activity factor (Australian identity) was significantly different between the two groups, with international students more likely to engage in these activities. The next section examines the relationships between travel motivations and tourism 
activity preferences using PLS using only the measurement items retained after the EFAs.

\section{Partial Least Squares Path Modeling (PLS-PM)}

Our preliminary analysis shows that the research data were nonnormal. Thus, PLS-PM was selected because it does not rely on the assumption of data normality and does not require as large a sample size as other causal modeling techniques (Arnett, Laverie, \& Meiers, 2003). The evaluation of the model involves a systematic examination of indices including $R^{2}$, average variance accounted for (AVA), average variance extracted (AVE), standardized loadings, path coefficients, and bootstrap critical ratios.

The outer model is a measurement model relating the manifest variables to their own latent variables
(Tenenhaus, Vinzi, Chatelin, \& Lauro, 2005). As Table 5 indicates, standardized factor loadings for all measurement items exceeded the requirement of 0.50 (Hair et al., 2006). In addition, all bootstrap critical ratios derived from the data were above the acceptable level (greater than 1.96, $p<0.05$ ) (Chin, 1998a) for all variables, suggesting that they are significant indicators of their respective constructs, thus providing support for convergent validity. Discriminant validity of the measured constructs was established as the AVE for each factor is greater than its squared correlations with other factors (Fornell \& Larcker, 1981), as Table 6 shows.

Construct reliability was evaluated with DillonGoldstein's (or Jöreskog's) rho (Chin, 1998b). As Table 5 shows, all factors achieved the recommended level of construct reliability $(\alpha>0.70)$

Table 2

Results for Exploratory Factor Analysis of Tourism Activities

\begin{tabular}{|c|c|c|c|c|c|c|}
\hline \multirow[b]{2}{*}{ Activity Factors and Items } & \multirow{2}{*}{$\begin{array}{l}\text { Variance } \\
\text { Explained }\end{array}$} & \multirow{2}{*}{$\begin{array}{c}\text { Reliability } \\
\alpha\end{array}$} & \multicolumn{4}{|c|}{ Factor Loading } \\
\hline & & & 1 & 2 & 3 & 4 \\
\hline Factor 1: Australian identity & $28.25 \%$ & 0.83 & & & & \\
\hline $\begin{array}{l}\text { Experiencing Aboriginal art/craft and } \\
\text { cultural displays }\end{array}$ & & & 0.79 & & & \\
\hline Visiting an aboriginal site/community & & & 0.78 & & & \\
\hline Visiting farms & & & 0.63 & & & \\
\hline Tourist trains & & & 0.63 & & & \\
\hline $\begin{array}{l}\text { Visiting museums or art galleries/art/ } \\
\text { craft workshops/studios }\end{array}$ & & & 0.55 & & & \\
\hline $\begin{array}{l}\text { Going whale/dolphin watching (in the } \\
\text { ocean) }\end{array}$ & & & 0.44 & & & \\
\hline Factor 2: Physical/outdoor & $10.61 \%$ & 0.82 & & & & \\
\hline Playing other sports & & & & 0.91 & & \\
\hline Attending an organized sporting events & & & & 0.66 & & \\
\hline Exercise/gym/swimming at a local pool & & & & 0.55 & & \\
\hline $\begin{array}{l}\text { Other outdoor activities (e.g., horse } \\
\text { riding, rock climbing, white water } \\
\text { rafting, bungee jumping, etc.) }\end{array}$ & & & & 0.54 & & \\
\hline Factor 3: Pleasure & $6.71 \%$ & 0.78 & & & & \\
\hline Eating out/restaurants & & & & & 0.91 & \\
\hline Going shopping (pleasure) & & & & & 0.66 & \\
\hline Day trip to another place & & & & & 0.55 & \\
\hline $\begin{array}{l}\text { Attending festivals/fairs or cultural } \\
\text { events }\end{array}$ & & & & & 0.54 & \\
\hline Visiting friends \& relatives & & & & & 0.42 & \\
\hline Factor 4: Nature-based & $5.81 \%$ & 0.79 & & & & \\
\hline Bushwalking & & & & & & 0.88 \\
\hline $\begin{array}{l}\text { Visiting national parks/botanical or } \\
\text { other public gardens }\end{array}$ & & & & & & 0.66 \\
\hline Rainforest walks & & & & & & 0.66 \\
\hline $\begin{array}{l}\text { Visiting history/heritage buildings, } \\
\text { sites or monuments }\end{array}$ & & & & & & 0.41 \\
\hline Total & $51.37 \%$ & & & & & \\
\hline
\end{tabular}


Table 3

Difference in Motivation Factors

\begin{tabular}{|c|c|c|c|c|c|c|c|}
\hline \multicolumn{2}{|l|}{ Motivation } & $\begin{array}{c}\text { Overall } \\
\text { Mean/Rank }\end{array}$ & \multicolumn{2}{|c|}{$\begin{array}{c}\text { International } \\
\text { Mean/Rank }\end{array}$} & $\begin{array}{l}\text { Australian } \\
\text { Mean/Rank }\end{array}$ & \multicolumn{2}{|c|}{$\begin{array}{c}\text { Independent Sample } \\
t \text { Test } p \text { Value }\end{array}$} \\
\hline Relax mentally & & $5.46 / 1$ & \multicolumn{2}{|c|}{$5.47 / 1$} & $5.44 / 1$ & \multicolumn{2}{|r|}{0.85} \\
\hline Relieve stress and tension & & $5.31 / 2$ & \multicolumn{2}{|c|}{$5.26 / 3$} & $5.35 / 2$ & \multicolumn{2}{|r|}{0.58} \\
\hline Rest & & $5.17 / 3$ & \multicolumn{2}{|c|}{$5.28 / 2$} & $5.03 / 3$ & \multicolumn{2}{|r|}{0.15} \\
\hline Discover new things & & $5.03 / 4$ & \multicolumn{2}{|c|}{$5.13 / 4$} & $4.94 / 4$ & \multicolumn{2}{|r|}{0.25} \\
\hline Relax physically & & $4.90 / 5$ & \multicolumn{2}{|c|}{$4.88 / 7$} & $4.90 / 5$ & \multicolumn{2}{|r|}{0.94} \\
\hline Expand my knowledge & & $4.85 / 6$ & \multicolumn{2}{|c|}{$5.12 / 5$} & $4.58 / 7$ & \multicolumn{2}{|r|}{0.00} \\
\hline $\begin{array}{l}\text { Meet new and different } \\
\text { people }\end{array}$ & & $4.84 / 7$ & \multicolumn{2}{|c|}{$5.11 / 6$} & $4.58 / 8$ & \multicolumn{2}{|r|}{0.00} \\
\hline $\begin{array}{l}\text { Be good at things I'm } \\
\text { interested in }\end{array}$ & & $4.61 / 8$ & \multicolumn{2}{|c|}{$4.82 / 8$} & $4.42 / 14$ & \multicolumn{2}{|r|}{0.02} \\
\hline Satisfy my curiosity & & $4.61 / 9$ & \multicolumn{2}{|c|}{$4.72 / 12$} & $4.50 / 10$ & \multicolumn{2}{|r|}{0.19} \\
\hline Learn things around me & & $4.60 / 10$ & \multicolumn{2}{|c|}{$4.78 / 9$} & $4.43 / 13$ & \multicolumn{2}{|r|}{0.03} \\
\hline Be active & & $4.60 / 11$ & \multicolumn{2}{|c|}{$4.74 / 11$} & $4.48 / 11$ & \multicolumn{2}{|r|}{0.12} \\
\hline Explore new ideas & & $4.57 / 12$ & \multicolumn{2}{|c|}{$4.77 / 10$} & $4.39 / 16$ & & 0.02 \\
\hline Interact with others & & $4.56 / 13$ & & & $4.63 / 6$ & & 0.47 \\
\hline $\begin{array}{l}\text { Improve my skills and } \\
\text { ability in doing them }\end{array}$ & & $4.54 / 14$ & & & $4.41 / 15$ & & 0.11 \\
\hline $\begin{array}{l}\text { Avoid the hustle and bustle } \\
\text { of daily activities }\end{array}$ & & $4.45 / 15$ & & & $4.58 / 9$ & & 0.16 \\
\hline Slow down & & $4.40 / 16$ & & & $4.46 / 12$ & & 0.49 \\
\hline Challenge my ability & & $4.35 / 17$ & & & $4.16 / 18$ & & 0.03 \\
\hline Be creative & & $4.28 / 18$ & & & $4.08 / 20$ & & 0.02 \\
\hline Build friendship with others & & $4.27 / 19$ & & & $4.15 / 19$ & & 0.11 \\
\hline Use my physical abilities & & $4.13 / 20$ & & & $4.21 / 17$ & & 0.36 \\
\hline Develop close friendship & & $3.90 / 21$ & & & $3.65 / 25$ & & 0.00 \\
\hline $\begin{array}{l}\text { Be socially competent and } \\
\text { skillful }\end{array}$ & & $3.89 / 22$ & & & $3.81 / 22$ & & 0.28 \\
\hline Keep in shape physically & & $3.89 / 23$ & & & $3.93 / 21$ & & 0.61 \\
\hline Learn about myself & & $3.79 / 24$ & & & $3.54 / 27$ & & 0.01 \\
\hline Develop physical abilities & & $3.78 / 25$ & & & $3.66 / 24$ & & 0.16 \\
\hline Use my imagination & & $3.75 / 26$ & & & $3.76 / 23$ & & 0.93 \\
\hline $\begin{array}{l}\text { Reveal my thought, } \\
\text { feelings, or physical } \\
\text { skills to others }\end{array}$ & & $3.64 / 27$ & & & $3.41 / 29$ & & 0.01 \\
\hline Gain a feeling of belonging & & $3.55 / 28$ & & & $3.33 / 31$ & & 0.01 \\
\hline Develop physical fitness & & $3.55 / 29$ & & & $3.57 / 26$ & & 0.90 \\
\hline Unstructure my time & & $3.42 / 30$ & & & $3.37 / 30$ & & 0.52 \\
\hline Be alone & & $3.38 / 31$ & & & $3.52 / 28$ & & 0.18 \\
\hline Gain other's respect & & $3.16 / 32$ & & & $3.05 / 32$ & & 0.20 \\
\hline & & verall & Intel & nal & Austı & & Independent \\
\hline Motivation Factors & Mean & Rank & Mean & Rank & Mean & Rank & Test $p$ Value \\
\hline Stimulus/avoidance & 5.05 & 1 & 5.05 & 1 & 5.04 & 1 & 0.94 \\
\hline Intellectual factor & 4.35 & 2 & 4.52 & 2 & 4.18 & 2 & 0.01 \\
\hline Social factor & 4.18 & 3 & 4.34 & 3 & 4.04 & 3 & 0.01 \\
\hline Competence/mastery & 4.18 & 4 & 4.27 & 4 & 4.10 & 4 & 0.21 \\
\hline
\end{tabular}

(Hair et al., 2006), with Dillon-Goldstein's rho values ranging from 0.85 to 0.90 , thus demonstrating construct reliability.

Table 7 shows the results of the inner model, with path coefficients between the exogenous and endogenous variables, the AVA, the $R^{2}$, and the critical ratios. The bootstrap critical ratios of the six path coefficients were greater than 1.96. Furthermore, as a measure of predictive relevance of the structural model, the AVA for the model was 
Table 4

Difference in Activity Factors

\begin{tabular}{|c|c|c|c|c|c|c|c|}
\hline \multirow{2}{*}{$\begin{array}{l}\text { Activity } \\
\text { Going to the beach (swimming, sunbathing, } \\
\text { surfing, diving etc.) }\end{array}$} & \multicolumn{2}{|c|}{$\begin{array}{c}\text { Overall } \\
\text { Mean/Rank }\end{array}$} & \multicolumn{2}{|c|}{$\begin{array}{c}\text { International } \\
\text { Mean/Rank }\end{array}$} & \multicolumn{2}{|c|}{$\begin{array}{l}\text { Australian } \\
\text { Mean/Rank }\end{array}$} & $\begin{array}{l}\text { Independent } \\
\text { sample } t \text { Test } \\
p \text { Value }\end{array}$ \\
\hline & \multicolumn{2}{|c|}{$5.75 / 1$} & \multicolumn{2}{|c|}{$5.69 / 1$} & \multicolumn{2}{|c|}{$5.80 / 1$} & 0.49 \\
\hline Eating out/restaurants & \multicolumn{2}{|c|}{$5.72 / 2$} & \multicolumn{2}{|c|}{$5.65 / 2$} & \multicolumn{2}{|c|}{$5.78 / 2$} & 0.39 \\
\hline Going shopping (pleasure) & \multicolumn{2}{|c|}{$5.31 / 3$} & \multicolumn{2}{|c|}{$5.27 / 5$} & \multicolumn{2}{|c|}{$5.34 / 3$} & 0.72 \\
\hline Visiting friends and relatives & \multicolumn{2}{|c|}{$5.27 / 4$} & \multicolumn{2}{|c|}{$5.22 / 7$} & \multicolumn{2}{|c|}{$5.33 / 4$} & 0.56 \\
\hline Visiting amusements/theme parks & \multicolumn{2}{|c|}{$5.26 / 5$} & \multicolumn{2}{|c|}{$5.24 / 6$} & \multicolumn{2}{|c|}{$5.27 / 5$} & 0.86 \\
\hline Day-trip to another place & \multicolumn{2}{|c|}{$5.24 / 6$} & \multicolumn{2}{|c|}{$5.21 / 8$} & \multicolumn{2}{|c|}{$5.27 / 6$} & 0.73 \\
\hline Attending festivals/fairs or cultural events & \multicolumn{2}{|c|}{$5.15 / 7$} & \multicolumn{2}{|c|}{$5.30 / 3$} & $5.01 /$ & & 0.10 \\
\hline $\begin{array}{l}\text { Other outdoor activities (e.g., horse riding, } \\
\text { rock climbing, rafting, bungee jump, etc.) }\end{array}$ & 5.01 & & 5.15 & & $4.88 /$ & & 0.15 \\
\hline $\begin{array}{l}\text { Just walking or driving around/general } \\
\text { sightseeing }\end{array}$ & 4.97 & & 5.09 & & $4.87 /$ & & 0.23 \\
\hline Watching movies/videos & 4.94 & & 4.88 & & $5.00 /$ & & 0.55 \\
\hline Picnics/BBQs & 4.90 & & 4.96 & & $4.84 /$ & & 0.49 \\
\hline Going whale/dolphin watching (in the ocean) & 4.90 & & 5.27 & & $4.54 /$ & & 0.00 \\
\hline Pubs, clubs, discos, etc. & 4.76 & & 4.30 & & $5.22 /$ & & 0.00 \\
\hline Visiting wildlife parks/zoos & 4.74 & & 5.01 & & $4.48 /$ & & 0.00 \\
\hline $\begin{array}{l}\text { Attending theatre/concerts or other } \\
\text { performing arts }\end{array}$ & 4.69 & & 4.82 & & $4.59 /$ & & 0.21 \\
\hline Going on guided tours or excursions & 4.65 & & 4.85 & & $4.45 /$ & & 0.04 \\
\hline Ferry rides, tall ships, other tourist cruises & 4.65 & & 4.88 & & $4.44 /$ & & 0.03 \\
\hline $\begin{array}{l}\text { Visiting history/heritage buildings, sites or } \\
\text { monuments }\end{array}$ & 4.63 & & 4.88 & & $4.38 /$ & & 0.01 \\
\hline $\begin{array}{l}\text { Visiting museums or art galleries/art/craft } \\
\text { workshops/studios }\end{array}$ & 4.62 & & 4.97 & & $4.27 /$ & & 0.00 \\
\hline Exercise/gym/swimming at a local pool & 4.55 & & 4.60 & & $4.50 /$ & & 0.62 \\
\hline Rainforest walks & 4.50 & & 4.49 & & $4.53 /$ & & 0.82 \\
\hline Playing other sports & 4.44 & & 4.66 & & $4.22 /$ & & 0.04 \\
\hline $\begin{array}{l}\text { Visiting national parks/botanical or other } \\
\text { public gardens }\end{array}$ & 4.43 & & 4.68 & & $4.20 /$ & & 0.01 \\
\hline Attending an organized sporting events & 4.37 & & 4.53 & & $4.21 /$ & & 0.11 \\
\hline Visiting the outback & 4.21 & & 4.54 & & $3.90 /$ & & 0.00 \\
\hline $\begin{array}{l}\text { Visiting industrial tourism attractions } \\
\text { (e.g., breweries, mines) }\end{array}$ & 4.17 & & 4.46 & & $3.88 /$ & & 0.00 \\
\hline Bushwalking & 4.11 & & 4.12 & & $4.12 /$ & & 0.98 \\
\hline Tourist trains & 4.02 & & 4.57 & & $3.47 /$ & & 0.00 \\
\hline Visiting wineries & 3.93 & & 4.22 & & $3.65 /$ & & 0.01 \\
\hline Visiting casinos & 3.92 & & 3.88 & & $3.95 /$ & & 0.71 \\
\hline Visiting farms & 3.84 & & 4.28 & & $3.41 /$ & & 0.00 \\
\hline Going fishing & 3.74 & & 3.75 & & $3.72 /$ & & 0.88 \\
\hline $\begin{array}{l}\text { Experiencing aboriginal art/craft and cultural } \\
\text { displays }\end{array}$ & 3.74 & & 4.42 & & $3.08 /$ & & 0.00 \\
\hline Visiting an aboriginal site/community & 3.60 & & 4.14 & & $3.07 /$ & & 0.00 \\
\hline Playing golf & 2.95 & & 2.97 & & $2.91 /$ & & 0.76 \\
\hline & Ov & & Intern & onal & Aust & ian & Independent \\
\hline Activity Factors & Mean & Rank & Mean & Rank & Mean & Rank & $p$ Value \\
\hline Pleasure & 5.34 & 1 & 5.3316 & 1 & 5.3451 & 1 & 0.916 \\
\hline Physical/outdoor & 4.59 & 2 & 4.7368 & 2 & 4.4493 & 2 & 0.081 \\
\hline Nature-based & 4.42 & 3 & 4.5395 & 4 & 4.3088 & 3 & 0.124 \\
\hline Australian identity & 4.12 & 4 & 4.6096 & 3 & 3.6383 & 4 & 0.000 \\
\hline
\end{tabular}


Table 5

Results for the Outer Model

\begin{tabular}{|c|c|c|c|c|c|}
\hline Latent Variable/Manifest Variables & $\begin{array}{l}\text { Standardized } \\
\text { Loading }\end{array}$ & SE & $\begin{array}{c}\text { Critical } \\
\text { Ratio }\end{array}$ & $\begin{array}{c}\text { Average } \\
\text { Variance } \\
\text { Extracted }\end{array}$ & $\begin{array}{c}\text { Dillion- } \\
\text { Goldstein } \\
\text { Rho }\end{array}$ \\
\hline \multicolumn{6}{|l|}{ INTEL } \\
\hline Learn about things around me & 0.75 & 0.04 & 20.78 & 0.55 & 0.89 \\
\hline Satisfy my curiosity & 0.72 & 0.04 & 19.63 & & \\
\hline Explore new ideas & 0.74 & 0.04 & 20.33 & & \\
\hline Learn about myself & 0.72 & 0.04 & 18.89 & & \\
\hline Expand my knowledge & 0.77 & 0.03 & 25.84 & & \\
\hline Be creative & 0.74 & 0.03 & 22.97 & & \\
\hline Use my imagination & 0.74 & 0.04 & 17.29 & & \\
\hline \multicolumn{6}{|l|}{ SOCIAL } \\
\hline Build friendship with others & 0.82 & 0.03 & 28.67 & 0.54 & 0.87 \\
\hline Interact with others & 0.76 & 0.04 & 18.30 & & \\
\hline Develop close friendship & 0.69 & 0.03 & 20.79 & & \\
\hline Meet new and different people & 0.80 & 0.03 & 25.16 & & \\
\hline Reveal my thoughts feelings, or physical skills to others & 0.68 & 0.06 & 12.25 & & \\
\hline Be socially competent and skillful & 0.61 & 0.06 & 10.12 & & \\
\hline \multicolumn{6}{|l|}{ COMP/MAST } \\
\hline Develop my abilities & 0.62 & 0.04 & 13.81 & 0.53 & 0.90 \\
\hline Be good in doing things I'm interested in & 0.62 & 0.05 & 12.02 & & \\
\hline Improve my skills and abilities in doing things I'm interested in & 0.71 & 0.04 & 20.24 & & \\
\hline Be active & 0.70 & 0.03 & 22.52 & & \\
\hline Develop physical skills and abilities & 0.79 & 0.03 & 26.74 & & \\
\hline Keep in shape physically & 0.78 & 0.04 & 20.49 & & \\
\hline Use my physical abilities & 0.76 & 0.03 & 26.54 & & \\
\hline Develop physical fitness & 0.80 & 0.02 & 34.42 & & \\
\hline \multicolumn{6}{|l|}{ STM/AVOID } \\
\hline Slow down & 0.72 & 0.04 & 18.80 & 0.57 & 0.87 \\
\hline Relax physically & 0.75 & 0.04 & 16.91 & & \\
\hline Relax mentally & 0.71 & 0.05 & 15.21 & & \\
\hline Rest & 0.82 & 0.03 & 29.66 & & \\
\hline Relieve stress and tension & 0.76 & 0.03 & 22.45 & & \\
\hline \multicolumn{6}{|l|}{ AUST ID } \\
\hline Go whale/dolphin watching & 0.55 & 0.05 & 9.98 & 0.53 & 0.88 \\
\hline Visiting farms & 0.71 & 0.05 & 15.60 & & \\
\hline Visiting museums or art galleries/art/craft workshops/studios & 0.72 & 0.04 & 19.43 & & \\
\hline Visiting an aboriginal site/community & 0.80 & 0.03 & 24.72 & & \\
\hline Experiencing aboriginal art/craft and cultural displays & 0.81 & 0.03 & 29.14 & & \\
\hline Tourist trains & 0.74 & 0.03 & 21.66 & & \\
\hline \multicolumn{6}{|l|}{$\mathrm{PHY} / \mathrm{OD}$} \\
\hline Other outdoor activities & 0.73 & 0.03 & 21.31 & 0.65 & 0.88 \\
\hline Playing other sports (other than golf) & 0.88 & 0.01 & 61.53 & & \\
\hline Exercise/gym/swimming at a local pool & 0.82 & 0.03 & 30.47 & & \\
\hline Attending an organized sports event & 0.78 & 0.03 & 27.32 & & \\
\hline \multicolumn{6}{|l|}{ PLEASURE } \\
\hline Attending festivals/fairs or cultural events & 0.77 & 0.03 & 23.60 & 0.54 & 0.85 \\
\hline Visiting friends \& relatives & 0.64 & 0.06 & 10.57 & & \\
\hline Going shopping & 0.72 & 0.05 & 13.50 & & \\
\hline Day trip to another place & 0.73 & 0.04 & 17.43 & & \\
\hline Eating out/restaurants & 0.80 & 0.03 & 30.06 & & \\
\hline \multicolumn{6}{|l|}{ NATURE } \\
\hline Visiting national parks/botanical or other public gardens & 0.83 & 0.03 & 30.57 & 0.61 & 0.87 \\
\hline Bushwalking & 0.82 & 0.03 & 26.44 & & \\
\hline Rainforest walks & 0.69 & 0.05 & 13.47 & & \\
\hline Visiting history/heritage buildings, sites or monuments & 0.76 & 0.04 & 19.67 & & \\
\hline
\end{tabular}

Standardized loadings, standard errors, and critical ratios are bootstrap estimates. 
Table 6

Discriminant Validity Analysis

\begin{tabular}{lcccccccc}
\hline & INTEL & SOCIAL & COMP/MAST & STM/AVOID & AUST ID & PHY/OD & PLEASURE & NATURE \\
\hline INTEL & $\mathbf{0 . 5 5}$ & & & & & & & \\
SOCIAL & 0.47 & $\mathbf{0 . 5 4}$ & & & & & & \\
COMP/MAST & 0.42 & 0.45 & $\mathbf{0 . 5 3}$ & & & & & \\
STM/AVOID & 0.10 & 0.06 & 0.08 & $\mathbf{0 . 5 7}$ & & & & \\
AUST ID & 0.14 & 0.10 & 0.10 & 0.04 & $\mathbf{0 . 5 3}$ & & & \\
PHY/OD & 0.09 & 0.10 & 0.27 & 0.03 & 0.11 & $\mathbf{0 . 6 5}$ & & \\
PLEASURE & 0.11 & 0.12 & 0.05 & 0.12 & 0.14 & 0.17 & $\mathbf{0 . 5 4}$ & $\mathbf{0 . 6 1}$ \\
NATURE & 0.13 & 0.05 & 0.08 & 0.08 & 0.30 & 0.04 & 0.07 & $\mathbf{0 . 0 4}$ \\
\hline
\end{tabular}

The bold diagonal elements are the variance shared between the constructs and their measures. Off-diagonal elements are the squared correlations between constructs. INTEL, intellectual; COMP/MAST, competence/mastery; STM/AVOID, stimulus/ avoidance; AUST ID, Australian identity; PHY/OD, physical/outdoor; NATURE, nature based.

0.206 (greater than the cutoff of 0.10 ) (Falk \& Miller, 1992), supporting the predictive power of the model and H1a to H1d.

To maximize the utility of the current study, we divided the overall sample into two groups, international and Australian, and conducted a multigroup analysis to examine the differences in path results for the two groups. The results show that all six paths are significant in the international and domestic subsamples. In addition, of the six paths tested, all are not statistically different across the two groups, with the exception of one (i.e., competence/mastery to physical/outdoor activities), which is stronger in the Australian group.

\section{Discussion and Implications}

\section{RQ1: Travel Motivations}

Stimulus/avoidance was the most important motivation at both the individual item and factor levels. In line with prior research (K. Kim \& Jogaratnam, 2003; X. Wang \& Walker, 2010), items highly motivating to students are all associated with relaxation. Such motivation was identified by Ryan (1998) as being at the bottom level of the travel career ladder. Escapism is commonly perused by tourists, but the results of this study seem to suggest its particular importance to university student travelers, who are likely to be inexperienced in travel planning and decision making because they have just grown into adulthood and gained autonomy in making travel decisions. In relation to the escape facet of stimulus avoidance, the everyday environment from which students want to escape is university life. Students tend to see travel as a break from study, whereas others with a fulltime job often have to undertake their studies during holiday time (Richards \& Wilson, 2004).

The escape facet of stimulus/avoidance (e.g., "to be alone," "to avoid the hustle and bustle of daily activities”) was less motivating to students. As X. Wang and Walker (2010) suggests, students generally

Table 7

Results for Inner Model

\begin{tabular}{|c|c|c|c|c|c|c|}
\hline Dependent Variable & $\begin{array}{l}\text { Predictor } \\
\text { Variables }\end{array}$ & Coefficient & SE & $\begin{array}{c}\text { Critical } \\
\text { Ratio }\end{array}$ & $R^{2}$ & Hypothesis \\
\hline AUST ID & INTEL & 0.382 & 0.047 & 8.012 & 0.148 & H1a \\
\hline $\mathrm{PHY} / \mathrm{OD}$ & COMP/MAST & 0.525 & 0.043 & 12.307 & 0.278 & $\mathrm{H} 1 \mathrm{c}$ \\
\hline \multirow[t]{2}{*}{ PLEASURE } & SOCIAL & 0.281 & 0.061 & 4.546 & \multirow[t]{2}{*}{0.211} & H1b \\
\hline & STM/AVOID & 0.291 & 0.055 & 4.924 & & H1d \\
\hline \multirow[t]{2}{*}{ NATURE } & INTEL & 0.306 & 0.076 & 3.984 & \multirow[t]{2}{*}{0.185} & $\mathrm{H} 1 \mathrm{a}$ \\
\hline & STM/AVOID & 0.205 & 0.061 & 3.130 & & H1d \\
\hline Average variance accounted & & & & & 0.206 & \\
\hline
\end{tabular}

Coefficients, SEs, critical ratios, and $R^{2}$ are bootstrap estimates. INTEL, intellectual; COMP/MAST, competence/mastery; STM/AVOID, stimulus/avoidance; AUST ID, Australian identity; PHY/OD, physical/outdoor; NATURE, nature based. 
experience less stress and pressure from their everyday social environment than they would after joining the workforce. This may also be the case with this study's sample. In addition, international students new to Australia may find the Australian educational experience rather novel and exciting, resulting in a lower desire to escape. In other words, motivation may be influenced by personal circumstance and experience with the destination.

\section{RQ2: Preferences for Activities}

In terms of preferences for activities, students particularly preferred leisure activities, including going to the beaches, going shopping, and eating out. Similar results were found in Xu et al. (2009) where both British students and their international counterparts showed a strong preference for the beach, highlighting opportunities for beach destinations such as the Gold Coast. Other likely choices were some highly rigorous outdoor activities, referred to as "adrenaline activities" (Richards \& Wilson, 2004), such as horse riding, rock climbing, whitewater rafting, and bungee jumping. However, these activities were not as popular among students in other countries (Field, 1999; Richards \& Wilson, 2004), a difference that may be worth exploring further to examine its underlying causes. These results are also reflected at the factor level, with pleasure and physical/outdoor activities being rated the most and second most preferred groups of activities. Some activities such as fishing, visiting wineries, and playing golf did not appeal to this group, a finding similar to a study conducted in the US (Field, 1999). These particular activities may be more appealing to the more mature travel segment. The findings of this study provide a basis for the industry to tailor marketing campaigns, and the industry is likely to gain the most benefit from concentrating on promoting popular activities.

\section{RQ3: Association Between Travel Motivations and Travelers' Preferred Activities}

In support of H1a to H1d, this study demonstrates a significant association between students' travel motivation and their preferences for activities. That is, students are more likely to engage in activities that satisfy their motivations. For instance, the stronger a student's competence/mastery motivation is, the more likely the student will be to participate in physical/outdoor activities that offer opportunities to develop skills and strength. Similarly, the more intellectually motivated a student is, the more likely he or she will be to engage in activities that offer opportunities to learn about Australia. Activities in the pleasure and nature categories allow students to relax and thus are positively associated with the motivation of stimulus/avoidance. The "biophilia effect" hypothesizes a deep affiliation between human beings and nature as a biological need, because being close to nature offers a range of psychological benefits (Lidwell, Holden, \& Butler, 2010). Therefore, nature-based activities can provide restorative benefits, such as stress and tension release, that satisfy the motivation of stimulus/avoidance. The social factor's significant relationship to activities in the pleasure category (e.g., visiting friends and relatives, eating out, attending festivals or cultural events) may be attributable to students' greater likelihood to participate in these activities with relatives and friends. The significant positive associations held true in both the domestic and international student subsamples. These results are in support of Moscardo et al.'s (1996) activitybased destination choice model.

\section{RQ4: Differences Between International and Domestic Students}

$\mathrm{H} 2 \mathrm{a}$ is supported, but international and domestic students' motivation differed mainly in relation to intellectual and social factors. International students were more likely to be motivated by new experiences such as learning new things and exploring new ideas. These activities can be seen as a pursuit of novelty, a crucial pull factor associated with destination attributes (Crompton, 1979). Two aspects of the sample may explain the stronger intellectual motivation. First, international students may have a greater deal of curiosity about Australia. Second, the greater maturity and the greater level of education of the international students may have contributed to their stronger intellectual motivation. Third, the sample is dominated by Asian students, especially Chinese, who are deeply influenced by Confucianism, in which learning is emphasized as an essential part of personal development (X. Wang 
\& Walker, 2010; Xu et al., 2009). In contrast, trips within Australia may not offer domestic students much sense of novelty and learning. This study also revealed that the social factor motivated international students significantly more than their Australian counterparts, as suggested by the results of the independent sample $t$ test. There is a stronger desire among international students for friendship and a sense of belonging than Australians. This is understandable given international students' less developed social network in Australia.

Supporting H2b, the two subsamples seem to differ in their preferences for activities, because 16 out of the 35 items are significantly different. However, at the factor level, difference exists in only one factor of Australian identity. Compared to domestic students, international students were more likely to engage in activities offering a learning experience about Australian culture such as attending festivals and cultural events, visiting historical and heritage buildings, and visiting museums or art galleries/art/ craft workshops/studios. This result corresponds to the earlier finding that international students were more motivated by the intellectual factor, and may again be explained by the dominance of Asian students in the sample. The two groups are also different in terms of the relative importance of activities. For example, high intensity activities, such as exercising, going to a gym, swimming at a local pool, rainforest walks, and bush walking, were more important to Australian students than to international students. Aboriginal attractions, which are iconic to Australian tourism, did not appeal much to international students, and even less to domestic students. Further studies are required to identify possible explanations for such differences.

\section{Theoretical and Practical Implications}

This study investigated university students' travel motivations and preferences for activities, adding to the limited literature relating to this important travel market. By identifying the differences between domestic and international students, this investigation highlights the heterogeneity of the student travel market. Further, the study shows the significant influence of travel motivation on tourism activity preferences, providing empirical evidence for the assertion that the relationship between motivation and destination choice is mediated through activities (Moscardo et al., 1996).

An understanding of the relationship between activity preference and tourist motivation is of paramount significance to destinations and tourism operators, because without it they are unable to design products that best satisfy tourist needs. For this reason, the results also have practical implications. First, the finding that stimulus/avoidance is an important motivation suggests that destinations offering relaxing environments and attractions can target the student market. Tour operators can also package products with relaxing attributes (e.g., hot springs, bars, and beaches) for this market. Second, the differences between international and domestic students require differentiated approaches to product design and marketing for the two market segments. For instance, given international students' higher score on intellectual motivation, tourism businesses marketing to these students need to emphasize the opportunity to learn and develop, and to increase the accessibility of these learning opportunities by, for example, providing free shuttle buses to museums and cultural events. Additionally, in light of international students' low confidence in their ability to communicate in English, operators must consider ways to minimize the language barrier (e.g., by offering brochures in different languages and using bilingual tour guides). Thirdly, the findings can serve as a reference for determining which activities would appeal to the university student market segment. Service providers can incorporate preferred activities identified in this study in their products. For instance, destinations could redesign and repackage Aboriginal attractions to cater to students' needs. One possibility would be to portray Aboriginal destinations as relaxed places that offer learning opportunities to satisfy both the relaxation and intellectual motivations of students.

\section{Limitations and Future Research Directions}

This study has several limitations. First, its cross-sectional research design determines that the results can only imply an association between the studied constructs rather than a causal relationship. Second, Asian students' dominance in the sample requires careful consideration when generalizing the results to students from other continents, such 
as Europe or South America. Another limitation lies in this study's focus on preference for activities rather than student travelers' actual participation in activities during real trips. One must be cautious when extrapolating from the results because preferences do not necessarily convert into actual behavior, which takes into account factors such as financial cost, time constraints, transport, and travel companions. Finally, factor means for both motivations and activity preferences were created on the basis of items retained after EFA, which may produce slightly different results compared to using the full set of measurement items.

Future studies can specifically include international students of other nationalities and regions, and consider the use of a longitudinal design to test the causal effect of motivations on travel activities. The difference between the student travel market and the wider youth market is another worthwhile topic for future investigation. Finally, from a theoretical point of view, future research can further clarify the possible mediating role of tourism activities between travel motivation and destination choice by including the whole motivation-activitydestination process.

\section{References}

Arnett, D. B., Laverie, D. A., \& Meiers, A. (2003). Developing parsimonious retailer equity indexes using partial least squares analysis: A method and applications. Journal of Retailing, 79(3), 161-170.

Australian Bureau of Statistics. (2012). Year Book Australia (No. 1301.0). Retrieved from http://www.abs.gov.au/ ausstats/abs@.nsf/mf/1301.0

Beard, J. G., \& Ragheb, M. G. (1983). Measuring leisure motivation. Journal of Leisure Research, 15(3), 219-228.

Chin, W. W. (1998a). Commentary: Issues and opinion on structural equation modeling. MIS Quarterly, 22(1), 7-16.

Chin, W. W. (1998b). The partial least squares approach to structural equation modelling. In G. A. Marcoulides (Ed.), Modern methods for business research (pp. 295336). Mahwah, NJ: Lawrence Erlbaum Associates.

Crompton, J. L. (1979). Motivations for pleasure vacation. Annals of Tourism Research, 6(4), 408-424.

Dann, G. (1977). Anomie, ego-enhancement and tourism. Annals of Tourism Research, 4(4), 184-194.

Falk, R. F., \& Miller, N. B. (1992). A primer for soft modeling. Akron, $\mathrm{OH}$ : University of Akron Press.

Field, A. M. (1999). The college student market segment: A comparative study of travel behaviors of international and domestic students at a southeastern university. Journal of Travel Research, 37(4), 375-381.

Fornell, C., \& Larcker, D. F. (1981). Evaluating structural equation models with unobservable variables and measurement error. Journal of Marketing Research, 18(1), 39-50.

Hair, J. F., Black, W. C., Babin, B. J., Anderson, R. E., \& Tatham, R. L. (2006). Multivariate data analysis. Upper Saddle River, NJ: Pearson Prentice Hall.

Hooper, K., \& van Zyl, M. (2011). Australia's tourism industry. Bulletin, Reserve Bank of Australia, December Quarter 2011. Retrieved February 19, 2013, from http:// www.rba.gov.au/publications/bulletin/2011/dec/pdf/ bu-1211-3.pdf

Hsieh, S., O’Leary, J. T., \& Morrison, A. M. (1992). Segmenting the international travel market by activity. Tourism Management, 13(2), 209-223.

Jackson, E. L., \& Schinkel, D. R. (1981). Recretional activity preferences of resident and tourist campers in the yellowknife region. Canadian Geographer, 25(4), 350-364.

Josiam, B. M., Smeaton, G., \& Clements, C. J. (1999). Involvement: Travel motivation and destination selection. Journal of Vacation Marketing, 5(2), 167-175.

Kim, C., \& Lee, S. (2000). Understanding the cultural differences in tourist motivation between Anglo-American and Japanese tourists. Journal of Travel \& Tourism Marketing, 9(1-2), 153-170.

Kim, K., \& Jogaratnam, G. (2003). Travel motivations: A comparative study of Asian international and domestic American college students. Journal of Travel \& Tourism Marketing, 13(4), 61-82.

Kim, K., Oh, I., \& Jogaratnam, G. (2007). College student travel: A revised model of push motives. Journal of Vacation Marketing, 13(1), 73-85.

Kim, K., Sun, J., \& Mahoney, E. (2008). Roles of motivation and activity factors in predicting satisfaction: Exploring the Korean cultural festival market. Tourism Analysis, 13(4), 413-425.

Kleiven, J. (2005). Measuring leisure and travel motives in Norway: Replicating and supplementing the leisure motivation scales. Tourism Analysis, 10(2), 109-122.

Law, R., Cheung, C., \& Lo, A. (2004). The relevance of profiling travel activities for improving destination marketing strategies. International Journal of Contemporary Hospitality Management, 16(6), 355-362.

Lidwell, W., Holden, K., \& Butler, J. (2010). Universal principles of design. Beverley, MA: Rockport Publishers.

Lounsbury, J. W., \& Franz, C. P. G. (1990). Vacation discrepancy: A leisure motivation approach. Psychological Reports, 66(2), 699-702.

Mansfeld, Y. (1992). From motivation to actual travel. Annals of Tourism Research, 19(3), 399-419.

Mazzarol, T., \& Soutar, G. N. (2002). "Push-pull” factors influencing international student destination choice. International Journal of Educational Management, 16(2), 82-90.

Moscardo, G., Morrison, A. M., Pearce, P. L., Lang, C. T., \& O’Leary, J. T. (1996). Understanding vacation destination 
choice through travel motivation and activities. Journal of Vacation Marketing, 2(2), 109-122.

Murray, E. J. (1964). Motivation and emotion. Englewood Cliffs, NJ: Prentice Hall.

Pearce, P., \& Son, A. (2004). Youth tourism markets in Australia: Comparing the travel behaviours of international English language students and backpackers. Tourism (Zagreb), 52(4), 341-350.

Richards, G., \& Wilson, J. (2004). The global nomad: Backpacker travel in theory and practice (Vol. 3). Cleveden, UK: Channel View Publications.

Ryan, C. (1998). The travel career ladder: An appraisal. Annals of Tourism Research, 25(4), 936-957.

Ryan, C., \& Glendon, I. (1998). Application of leisure motivation scale to tourism. Annals of Tourism Research, 25(1), 169-184.

Sakakida, Y., Cole, S. T., \& Card, J. A. (2004). A crosscultural study of college students' travel preferences: A value-oriented perspective. Journal of Travel \& Tourism Marketing, 16(1), 35-41.

Shoham, A., Schrage, C., \& van Eeden, S. (2005). Student travel behavior: A cross-national study. Journal of Travel \& Tourism Marketing, 17(4), 1-10.

Smith, S. L., \& Godbey, G. C. (1991). Leisure, recreation and tourism. Annals of Tourism Research, 18(1), 85-100.

Tabachnick, B. G., \& Fidell, L. S. (2001). Using multivariate statistics. Upper Sandle River, NJ: Pearson Education.

Tangeland, T., Vennesland, B., \& Nybakk, E. (2012). Second-home owners' intention to purchase nature-based tourism activity products: A Norwegian case study. Tourism Management, 36, 364-376.

Tapachai, N., \& Waryszak, R. (2000). An examination of the role of beneficial image in tourist destination selection. Journal of Travel Research, 39(1), 37-44.

Tenenhaus, M., Vinzi, V. E., Chatelin, Y. M., \& Lauro, C. (2005). PLS path modelling. Computational Statistics \& Data Analysis, 48, 159-205.

Trauer, B. (2006). Conceptualizing special interest tourism-frameworks for analysis. Tourism Management, 27(2), 183-200.

Wang, X., \& Walker, G. J. (2010). A comparison of Canadian and Chinese university students' travel motivations. Leisure/Loisir, 34(3), 279-293.

Wang, Y., \& Davidson, M. C. (2008). Chinese student travel market to Australia: An exploratory assessment of destination perceptions. International Journal of Hospitality \& Tourism Administration, 9(4), 405-426.

Wang, Y., \& Davidson, M. C. (2009). Chinese leisure tourists: Perceptions and satisfaction with Australia. Tourism Analysis, 14(6), 737-747.

Weaver, D. B. (2003). The contribution of international students to tourism beyond the core educational experience: Evidence from Australia. Tourism Review International, 7(2), 95-105.

Xu, F., Morgan, M., \& Song, P. (2009). Students' travel behaviour: A cross-cultural comparison of UK and China. International Journal of Tourism Research, 11(3), 255-268. 